\title{
ENDOMORPHISM NEAR-RINGS
}

\author{
by J. J. MALONE and C. G. LYONS \\ (Received 3rd March 1969)
}

The study of near-rings is motivated by consideration of the system generated by the endomorphisms of a (not necessarily commutative) group. Such endomorphism near-rings also furnish the motivation for the concept of a distributively generated (d.g.) near-ring. Although d.g. near-rings have been extensively studied, little is known about the structure of endomorphism near-rings. In this paper results are presented which enable one to give the elements of the endomorphism near-ring of a given group. Also, some results relating to the right ideal structure of an endomorphism near-ring are presented. These concepts are applied to present a detailed picture of the properties of the endomorphism near-ring of $\left(S_{3},+\right)$.

\section{Preliminaries}

A near-ring is a triple $(R,+,$.$) such that (R,+)$ is a group, $(R,$.$) is a semi-$ group, and . is left distributive over + ; i.e. $w(x+z)=w x+w z$ for each $w, x, z \in R$. A near-ring $R$ is d.g. if there exists $S \subset R$ such that $(S,$.$) is a sub-$ semigroup of $(R,$.$) , each element of S$ is right distributive, and $S$ is an additive generating set for $(R,+)$. The near-ring generated additively by all the endomorphisms of a (not necessarily commutative) group $(G,+)$ is d.g., $S$ being the set of endomorphisms. Such a near-ring will be called an endomorphism near-ring and will be denoted by $E(G)$.

A subset $K$ of a near ring $R$ is an ideal if $(K,+)$ is a normal subgroup of $(R,+), r k \in K$, and $\left(r_{1}+k\right) r_{2}-r_{1} r_{2} \in K$ for each $r, r_{1}, r_{2} \in R$ and $k \in K$. A. Fröhlich (5) has noted that for d.g. near-rings the third condition is equivalent to $k r \in K$. A subset $K$ is a right (left) ideal if $K$ satisfies the first and third (second) conditions.

Fröhlich $((6), 2.4)$ has shown that the near-ring generated by all the inner automorphisms of a finite simple, non-commutative, group $(G,+)$ is $E(G)$. In fact, this near-ring generated by the inner automorphisms consists of all the mappings of $G$ into $G$ which leave 0 fixed. A. J. Chandy (4) has given a necessary and sufficient condition that the near-ring generated by the inner automorphisms of a group be a ring. However, the more general endomorphism near-ring has not been studied.

If $\alpha$ is an endomorphism of $(G,+)$ and $g \in G$, the image of $g$ under $\alpha$ is denoted by $g \alpha$. Addition of functions on $G$ is done pointwise and multiplication of such functions is composition. 


\section{Properties of $E(G)$}

The following theorem is due to G. Berman and R. J. Silverman ((2), p. 27).

Theorem 2.1. Let e be an idempotent in the near-ring $R$. Then each $r \in R$ has two unique decompositions $r=(r-e r)+e r=e r+(-e r+r)$. Thus

where

$$
R=A_{e}+M_{e}=M_{e}+A_{e}
$$

and $A_{e} \cap M_{e}=0$.

The next theorem is immediate.

Theorem 2.2. $A_{e}$ is a right ideal of $R$. If $0 r=0$ for each $r \in R$, then $A_{e}$ is also a subnear-ring of $R . \quad M_{e}$ is a subnear-ring of $R$.

Fröhlich ((5), p. 80) showed that a d.g. near-ring satisfies the $0 r=0$ condition.

Theorem 2.3. Let $R$ be a near-ring such that $(R,+)$ is generated by $\left\{r_{\gamma} \mid \gamma \in \Gamma\right.$, an index set $\}$. Then $A_{e}$ is the normal subgroup generated by $\left\{r_{\gamma}-e r_{\gamma} \mid \gamma \in \Gamma\right\}$ and $M_{e}$ is the subgroup generated by $\left\{e_{\gamma} \mid \gamma \in \Gamma\right\}$.

Proof. Let $r \in R$. Then $r$ can be written as $r=c_{1}+c_{2}+\ldots+c_{k}$, with either $c_{i}$ or $-c_{i}$ in $\left\{r_{\gamma} \mid \gamma \in \Gamma\right\}, i=1, \ldots, k$. Several cases arise. The proof is presented for the case in which each $c_{i} \in\left\{r_{\gamma} \mid \gamma \in \Gamma\right\}$. No additional difficulties arise in the other cases. The theorem follows from

$$
\begin{aligned}
r= & c_{1}+c_{2}+\ldots+c_{k}=\left(\left(c_{1}-e c_{1}\right)+e c_{1}\right)+\left(\left(c_{2}-e c_{2}\right)+e c_{2}\right) \\
& +\ldots+\left(\left(c_{k}-e c_{k}\right)+e c_{k}\right)=\left[c_{1}-e c_{1}\right]+\left[e c_{1}+\left(c_{2}-e c_{2}\right)-e c_{1}\right] \\
& +\left[e c_{1}+e c_{2}+\left(c_{3}-e c_{3}\right)-e c_{2}-e c_{1}\right] \\
& +\ldots+\left[e c_{1}+e c_{2}+\ldots+e c_{k-1}+\left(c_{k}-e c_{k}\right)-e c_{k-1}-\ldots-e c_{2}-e c_{1}\right] \\
& +\left(e c_{1}+e c_{2}+\ldots+e c_{k}\right) .
\end{aligned}
$$

Corollary 2.4. Let $R$ be d.g. and e right distributive. Then $M_{e}$ is d.g.

Proof. Let $S=\left\{r_{\gamma} \mid \gamma \in \Gamma\right\}$. Then $(x+z) e r_{y}=(x e+z e) r_{\gamma}=x e r_{\gamma}+z e r_{\gamma}$, for each $x, z \in R$ and each $\gamma \in \Gamma$. So $M_{e}$ is d.g. with generating set $\left\{e r_{\gamma} \mid \gamma \in \Gamma\right\}$.

It is known ((7), p. 48) that a subnear-ring of a d.g. near-ring need not be d.g. It is shown below that even for an $E(G)$ there is no assurance that either $A_{e}$ or $M_{e}$ is d.g. However, Corollary 2.4 tells us that for a properly selected $e, M_{e}$ is d.g. In the example of $E\left(S_{3},+\right)$ to be considered below, $A_{e}$ will be d.g. if $e$ is right distributive. For an arbitrary $G$ it is unknown if $e$ right distributive implies that $A_{e}$ is distributively generated.

The following result points the way to some of the right ideals in $E(G)$. The proof is immediate. 
Theorem 2.5. Let $T$ be a non-empty subset of the group $G$. Let

$$
K=\{\alpha \in E(G) \mid T \alpha=0\} .
$$

If $K$ is non-empty, $K$ is a right ideal in $E(G)$.

\section{The elements of $E\left(S_{3},+\right)$}

Let $(S,+)$ designate the non-abelian group of order six with addition as given in Table I. In this section the elements of $E(S)$ will be displayed.

\begin{tabular}{c|llllll}
\multicolumn{7}{|c}{ TABLE I } \\
+ & 0 & $a$ & $b$ & $c$ & $d$ & $e$ \\
\hline 0 & 0 & $a$ & $b$ & $c$ & $d$ & $e$ \\
$a$ & $a$ & 0 & $e$ & $d$ & $c$ & $b$ \\
$b$ & $b$ & $d$ & 0 & $e$ & $a$ & $c$ \\
$c$ & $c$ & $e$ & $d$ & 0 & $b$ & $a$ \\
$d$ & $d$ & $b$ & $c$ & $a$ & $e$ & 0 \\
$e$ & $e$ & $c$ & $a$ & $b$ & 0 & $d$
\end{tabular}

Since $S$ is finite each element of $E(S)$ can be expressed as a (finite) sum of endomorphisms of $S$. It follows that each function in $E(S)$ maps 0 to 0 . Each function in $E(S)$ can then be represented by a 5-tuple: the first co-ordinate being the image of $a$, the second the image of $b$, etc. For instance, the 5-tuple (abcde) represents the identity function.

Since the only non-trivial normal subgroup of $S$ is $\{0, d, e\}$ and since the only automorphisms of $S$ are the inner automorphisms, it follows that there are exactly ten endomorphisms of $S$. Among these is the idempotent endomorphism (aaa00). Using the idempotent $\alpha=(a a a 00)$ we apply Theorem 2.3 to determine the elements of $E(S)$. Of course, the set of endomorphisms is our generating set. The endomorphisms and their decompositions (in the sense of Theorem 2.1) are given in Table II.

From Table II and Theorem 2.3 it follows that $M_{\alpha}=\{(x \times 00) \mid x \in S\}$ while $A^{\prime}$, the group generated by $\left\{r_{\gamma}-\alpha r_{\gamma}\right\}$, is $\{(00000)$, (0dede), (0eded $\left.)\right\}$. It is clear that $A^{\prime}$ is not normal:

$$
(a a a 00)+(0 e d e d)-(a a a 00)=(0 \text { deed }) .
$$

Consider the group generated by $A^{\prime}$ and (0deed). In this group we have

$$
(000 d e)=(0 \text { deed })+(0 e d e d) \text { and }(0 \text { de00 })=(0 e d d e)+(0 e d e d) .
$$

In fact, the group generated by $A^{\prime}$ and ( 0 deed) is

$$
A=((0 d e 00)) \oplus((000 d e))
$$

where $(\lambda), \lambda \in E(G)$, is the subgroup generated by $\lambda$. For any function in $E(S)$ the fourth and fifth coordinates must be $00, d e$, or $e d$. Thus $((000 d e))$ is a normal subgroup of $E(S)$. The subgroup ((0de00)) is invariant under conjugation by 
elements of $M_{\alpha}$ and is a normal subgroup of $A$. Thus $((0 d e 00))$ is normal in the subgroup generated by $A$ and $M_{\alpha}$. But then $A$, as a sum of normal subgroups, is normal in the group generated by $A$ and $M_{\alpha}$ and $A+M_{\alpha}=M_{\alpha}+A$. Thus

TABLE II

$\begin{array}{ccc}r_{\gamma} & \alpha r_{\gamma} & r_{\gamma}-\alpha r_{\gamma} \\ (00000) & (00000) & (00000) \\ (a a a 00) & (a a a 00) & (00000) \\ (b b b 00) & (b b b 00) & (00000) \\ (c c c 00) & (c c c 00) & (00000) \\ (a b c d e) & (a a a 00) & (0 d e d e) \\ (\text { acbed }) & (a a a 00) & (0 e d e d) \\ (c b a e d) & (c c c 00) & (0 e d e d) \\ (\text { baced }) & (b b b 00) & (0 e d e d) \\ \text { (cabde) } & (c c c 00) & (0 d e d e) \\ \text { (bcade) } & (b b b 00) & (0 d e d e)\end{array}$

TABLE III

An $I$ indicates an idempotent element. An $N$ indicates a nilpotent element.

\begin{tabular}{|c|c|c|c|}
\hline Order Two & Order & Three & Order Six \\
\hline$I(a a a 00)$ & $I(000 d e)$ & $I \quad(d 0 e d e)$ & $I(a b c d e)$ \\
\hline$I(b b b 00)$ & $(000 e d)$ & (e0ded) & (acbed) \\
\hline$I(c c c 00)$ & $I($ dddde $)$ & $I(e d 0 d e)$ & (cbaed) \\
\hline$I(a b c 00)$ & (eeeed) & (de0ed) & (baced) \\
\hline$(a c b 00)$ & $I$ (eeede) & $N(0 e d 00)$ & (cabde) \\
\hline$($ bac 00$)$ & (ddded) & $N(0 d e 00)$ & (bcade) \\
\hline$(b c a 00)$ & $I$ (Odede) & $N(d e 000)$ & $I$ (aaade) \\
\hline$(c a b 00)$ & (0eded) & $N(e d 000)$ & $I(b b b d e)$ \\
\hline$(c b a 00)$ & $I(e 0 d d e)$ & $N(d 0 e 00)$ & $I(c c c d e)$ \\
\hline & $(d 0 e e d)$ & $N(e 0 d 00)$ & (acbde) \\
\hline & $I(d e 0 d e)$ & $N(d d d 00)$ & (cbade) \\
\hline & $(e d 0 e d)$ & $N($ eee 00$)$ & (bacde) \\
\hline & I (0edde) & & (bcaed) \\
\hline & (0deed) & & (abced) \\
\hline & & & (cabed) \\
\hline & & & (aaaed) \\
\hline$I, N(00000)$ & & & (bbbed) \\
\hline & & & (ccced) \\
\hline
\end{tabular}

$E(S)=A+M_{\alpha}$ and $A$ is $A_{\alpha}$. Since $\left|A_{\alpha}\right|=9$ and $\left|M_{\alpha}\right|=6,|E(S)|=54$. The elements of $E(S)$, classified according to additive orders, are given in Table III. 
By Corollary 2.4 the subnear-ring $M_{\alpha}$ is d.g. It is also to be noted that $\left(M_{\alpha},+\right) \cong(S,+)$. The near-ring generated by $(0 d e 00)$ has the null multiplication (every product is $(00000)$ ) and thus is a ring. The near-ring generated by $(000 d e)$ is a field of order three. It follows that the right ideal $A_{\alpha}$ is a ring.

The non-zero elements of $E(S)$ have order two, three, or six. The elements of order three and $(00000)$ form a group of order twenty-seven. This group, having index two, is normal and thus, as a Sylow subgroup, is the only group of order twenty-seven. Also, it is a maximal subgroup.

\section{The ideal structures of $E(S)$}

In this section the right (left) (two-sided) ideals of $E(S)$ and the radical of $E(S)$ will be determined. We start with the observation that the subgroups of order two in $E(S)$ are non-unique Sylow subgroups. So no one of them is normal and there are no right (left) ideals of order two.

An application of Theorem 2.5 yields the annihilating right ideals as given in Table IV. The appropriate $T$ is listed with each of these right ideals.

\section{TABLE IV}

$$
\begin{aligned}
& T=\{c, d, e\}, \quad I_{1}(3)=((d e 000)) \\
& T=\{b, d, e\}, \quad I_{2}(3)=((d 0 e 00)) \\
& T=\{a, d, e\}, \quad I_{3}(3)=((0 d e 00)) \\
& T=\{a, b, c\}, \quad I_{4}(3)=((000 d e)) \\
& T=\{c\}, \quad I_{1}(9)=I_{1}(3) \oplus I_{4}(3) \\
& T=\{b\}, \quad I_{2}(9)=I_{2}(3) \oplus I_{4}(3) \\
& T=\{a\}, \quad I_{3}(9)=I_{3}(3) \oplus I_{4}(3) \\
& T=\{d, e\}, \quad I(18)=\left\{\lambda \in E(S) \mid 2 \lambda=(00000) \text { or } \lambda^{2}=(00000)\right\}
\end{aligned}
$$

Table IV lists four right ideals of order three. $I_{1}(3), I_{2}(3)$, and $I_{3}(3)$ have the null multiplication, $I_{4}(3)$ is a field of order three. Upon checking Table III we find that $E(S)$ has five normal subgroups of order three. The subgroup not so far considered is $((d d d 00))$. This subgroup determines a right ideal $I_{5}(3)$ which has the null multiplication. Thus, the five right ideals of order three are rings. Of the five, only $I_{5}(3)$ is also a left ideal and so is the unique (left) ideal of order three.

To the three right ideals of order nine listed in Table IV we may add

and

$$
I_{4}(9)=I_{1}(3) \oplus I_{2}(3)=I_{1}(3) \oplus I_{3}(3)=I_{2}(3) \oplus I_{3}(3)
$$

$$
I_{5}(9)=I_{4}(3) \oplus I_{5}(3) \text {. }
$$

Using a technique similar to that used in Section 3 in treating $A^{\prime}$ and $A$, we see that $E(S)$ has only these five normal subgroups of order nine. Of these, only $I_{4}(9)$ is also a left ideal and so is the unique (left) ideal of order nine. $I_{4}(9)$ has the null multiplication. Each right (left) ideal of order nine is a ring. 
Since any function in $E(S)$ carries the pair $d e$ to $00, d e$, or $e d$, it is seen that the subgroup of order twenty-seven determines a right (left) ideal (call it I(27)) which is unique and maximal. Noting that

$$
I(27)=I_{1}(3) \oplus I_{3}(3) \oplus I_{4}(3)
$$

we see that $I(27)$ is a ring with right identity $(000 \mathrm{de})$.

Each element of $I(18)$ carries the pair de to 00 . It is immediate that $I(18)$ is a maximal right (left) ideal. In Corollary 4.3 it will be verified that $I(18)$ is unique as a right (left) ideal. In Section 5 it will be shown that $I(18)$ is not a d.g. near-ring.

The proof of the following proposition is immediate.

Proposition 4.1. 1. In $E(S)$, the elements of order two not of the form (xxx00) form a multiplicative group $M$.

2. In $E(S)$, the conjugate of an element of order two of the form $(x x x 00)$ by (abc00) is in $M$.

4.1.1 implies that any right (left) ideal containing an element of order two not of the form $(x x x 00)$ must contain $M$ and has order $\geqq 7$. 4.1.2 implies that any right (left) ideal containing an element of order two of the form $(x x \times 00)$ must contain $M$ and thus must have order $\geqq 8$. Thus a proper right (left) ideal which contains an element of order two has order 18. Also, a proper right (left) ideal which contains an element of order six has order 18. Hence there are no right (left) ideals of order six.

Proposition 4.2. A proper right (left) ideal of $E(S)$ containing an element of order two consists of functions which map the pair de to 00.

Proof. Let $A$ be a proper right (left) ideal. Let $\delta, \lambda \in A$ with $|\delta|=2$ and $\{d, e\} \lambda \neq 0$. Either $|\lambda|=6$ or $|\lambda|=3$. If $|\lambda|=6,2 \lambda=(000 d e)$ or $(000 e d)$. If $|\lambda|=3$, then $|\lambda+\delta|=6$. In any case, $(000 d e) \in A$. By Proposition 4.1, $(a b c 00) \in A$. Therefore the identity map $(a b c d e) \in A$ and $A=R$. The result follows from this contradiction.

Corollary 4.3. I(18) is the only right (left) ideal of order eighteen.

Proof. I(18) contains all functions sending de to 00 .

Several authors have characterized the radical of a near-ring (for instance, (1) and (3)). This radical is the analogue of the Jacobson radical of ring theory and has the usual radical properties.

Definition 4.4. A subgroup $H$ of the near-ring $R$ is an $R$-subgroup if $H R \subset H$. The radical $J(R)$ is the intersection of the right ideals of $R$ which are maximal $R$-subgroups.

Theorem 2.2 of (1) is of particular interest with regard to $E(S)$ for it states that if $R$ is a finite d.g. near-ring with identity whose additive group $(R,+)$ is solvable, then $J(R)$ is nilpotent and the quotient near-ring $R / J(R)$ is a ring. It is easily seen that $(E(S),+)$ is solvable. 
Since the right ideals of order three or nine of $E(S)$ are subsets of $I(27)$, since there are no right ideals of order two or six, and since $I(18)$ and $I(27)$ are then the maximal $E(S)$-subgroups, $J(E(S))=I(18) \cap I(27)$, i.e. $J(E(S))=I_{4}(9)$. Thus, $I_{\mathbf{4}}(9)$ is a two-sided ideal.

Proposition 4.5. Let $L$ be a left ideal of $E(S)$. Let $\lambda \in L$, with $|\lambda|=3$, and let $\lambda$ map the pair to either de or ed. Then $(d e 000),(d 0 e 00) \in L$. That is, $J(E(S)) \subset L$.

From this proposition we see that the radical is the unique left ideal of order nine.

It is to be noted that the near-ring $E(S) / J(E(S))$ is isomorphic to the subnearring generated by ( $a b c d e$ ) and that each of these is isomorphic to the ring of integers modulo six.

$J(E(S))$ is the set of all nilpotent elements of $E(S)$. In fact, $J(E(S))$ has the null multiplication.

\section{Comments}

The only elements of $I(18)$ which are right distributive in $I(18)$ are the four endomorphisms contained in $I(18)$. These four elements do not constitute an additive generating set for $I(18)$. No other element of $I(18)$ is right distributive since all others, except $(d e 000)$ and $(e d 000)$, fail to distribute over the sum $(a a a 00)+(b b b 00)$ and $(d e 000)$ and $(e d 000)$ fail to distribute over the sum $(a b c 00)+(a b c 00)$. Thus $I(18)$ is not d.g. So we have a new example showing that a subnear-ring of a d.g. near-ring need not be d.g. The only other example appearing in the literature is that noted in (7). In that example the subnearring is not a right ideal.

In contrast to the situation of Corollary 2.4, we can show that $M_{e}$ need not be d.g. if $e$ is not right distributive. For $\beta=(a b c 00), M_{\beta}=I(18)$. But, as we have seen, $I(18)$ is not d.g. If we let $\gamma=(000 d e)$, then $A_{\gamma}=I(18)$ and we see that an $A_{e}$ need not be d.g. One would like to determine the precise relation between $e$ being right distributive and $A_{e}$ and $M_{e}$ being d.g. subnear-rings of $E(G)$.

Our example of $E(S)$ also leads to the formulation of the following conjecture: The near-ring $E(G)$ contains an idempotent element $e$ such that

$$
\left(M_{e},+\right) \cong(G,+) \text {. }
$$

In subsequent papers, further properties of $E(G)$ will be explored and additional examples will be presented. It will be shown, by using the technique of Theorem 2.3, that $E\left(D_{8}\right)$ has 256 elements.

\section{REFERENCES}

(1) J. C. Beidleman, Distributively generated near-rings with descending chain condition, Math. Z. 91 (1966), 65-69. 
(2) G. Berman and R. J. Silverman, Near-rings, Amer. Math. Monthly 66 (1959), 23-34.

(3) G. BetsCh, Ein Radikal für Fastringe, Math. Z. 78 (1962), 86-90.

(4) A. J. CHANDY, Rings generated by inner automorphisms of non-abelian groups, Doctoral Dissertation, Boston University, 1965.

(5) A. FröHLICH, Distributively generated near-rings, Proc. London Math. Soc. (3) 8 (1958), 76-108.

(6) A. FröHLICH, The near-ring generated by the inner automorphisms of a finite simple group, J. London Math. Soc. 33 (1958), 95-107.

(7) H. E. Heatherly, Embedding of near-rings, Doctoral Dissertation, Texas A\&M University, 1968.

(8) V. Tharmaratnam, Complete primitive distributively generated near-rings, Quart. J. Math. Oxford Ser. (2) 18 (1967), 293-313.

DepartMENT OF Mathematics

TEXAS A\&M UNIVERSITY

College Station, Texas 77843 